\title{
Coexistence or a related condition: an infant with retinoblastoma and Gaucher disease
}

\author{
Burcu Berberoğlu-Ateş ${ }^{1}$, Ali Varan², Hülya Demir ${ }^{1}$, Canan Akyüzz ${ }^{2}$, Aysel Yüce ${ }^{1}$ \\ Departments of ${ }^{1}$ Pediatric Gastroenterology, Hepatology and Nutrition and ${ }^{2}$ Pediatric Oncology, Hacettepe University \\ Faculty of Medicine, Ankara, Turkey.E-mail: burcuberber@hotmail.com \\ Received: 12th September 2018, Accepted: 23rd September 2018
}

SUMMARY: Berberoğlu-Ateş B, Varan A, Demir H, Akyüz C, Yüce A. Coexistence or a related condition: an infant with retinoblastoma and Gaucher disease. Turk J Pediatr 2019; 61: 449-452.

Gaucher disease (GD) is the most prevalant lysosomal lipid storage disease that results from loss of function of acid $\beta$-glucosidase due to mutations in the glucocerebrosidase gene. Common features of all types of GD include hepatosplenomegaly, cytopenia, and various patterns of bone and lung involvement.

Retinoblastoma is a malignant tumor of the developing retina that occurs in children, typically before the age of five. Retinoblastoma develops from cells that have cancer-predisposing variants in both copies of RB1.

The association between GD and retinoblastoma has not been reported until now. Here we report the case that was diagnosed with, retinoblastoma at the age of 2 months and then GD at the age of 11 months.

Although there are controversies concerning the association between GD and cancer; malignancies should be kept in mind during GD patients follow up.

Key words: Gaucher disease, retinoblastoma, cancer.

Gaucher disease is a rare inherited lysosomal storage disorder that is caused by the mutations in the gene for the enzyme glucocerebrosidase (GBA1). Accumulation of glucocerebroside in the lysosomes of macrophages results in hepatosplenomegaly, cytopenia, growth retardation, bone disease (pain, bone crises, osteoporosis, avascular necrosis) and depending on the type, neurological involvement. It has been reported that the incidence of malignant disorders is increased in patients with GD. ${ }^{1,2}$ However some studies indicate that except for multiple myeloma the incidence of solid tumors and hematologic malignancies are not significantly increased..$^{3,4}$

Retinoblastoma is the most common primary intraocular malignancy of childhood. It is a disease of infants and toddlers, with $95 \%$ of cases occurring before the age of 5 years. ${ }^{5}$ The disease is most likely to be originated from the precursors of cone photoreceptors. Incidence of retinoblastoma is constant worldwide at one case per 15 000-20 000 live-birth. ${ }^{6}$

The association between GD and retinoblastoma has not been reported until now. Here we report the case that was diagnosed retinoblastoma at the age of 2 months and then GD at the age of 11 months.

\section{Case Report}

An 11-month-old infant was referred to our hospital with complaint of abdominal distention. He had been diagnosed with bilateral retinoblastoma at the age of 2 months and unilateral enucleation was performed on

This study was presented at the $5^{\text {th }}$ Congress of Lysosomal Disorders with International Participation, 14-17 April 2016, Bodrum, Turkey. 
his right eye. Standard chemotherapy protocol for retinoblastoma was given 6 times for his left eye during his follow up period. He was born to consanguineous parents.

On admission, physical examination revealed a mildly malnourished patient with hepatosplenomegaly (spleen $8 \mathrm{~cm}$, liver was $6 \mathrm{~cm}$ palpable below the subcostal margin). Laboratoryvalues confirmed thrombocytopenia (platelets $71000 / \mu \mathrm{l}$ ). An iliac crest bone marrow aspiration sample demonstrated infiltration of lipid-laden foamy macrophages (Fig. 1). Serum level of chitotriosidase was markedly increased $(4208 \mathrm{nmol} / \mathrm{ml} / \mathrm{h}$; normal $<78.5 \mathrm{nmol} / \mathrm{hr} / \mathrm{ml}$ ) and glucocerebrosidase enzyme activity was decreased $(1.16 \mathrm{nmol} / \mathrm{h} /$ $\mathrm{mg}$ protein, reference ranges; $9.4 \pm 3.2 \mathrm{nmol} / \mathrm{h} /$ $\mathrm{mg}$ protein). GBA1 gene analysis revealed that he was homozygous for the L444P point mutation. Enzyme replacement therapy was initiated with imiglucerase (Cerezyme ${ }^{\circledR}, 50$ $\mathrm{U} / \mathrm{kg}$ body weight every 2 weeks). At the end of the 40th week of treatment, we observed decrement of the liver and spleen volumes and improvement in platelet count.

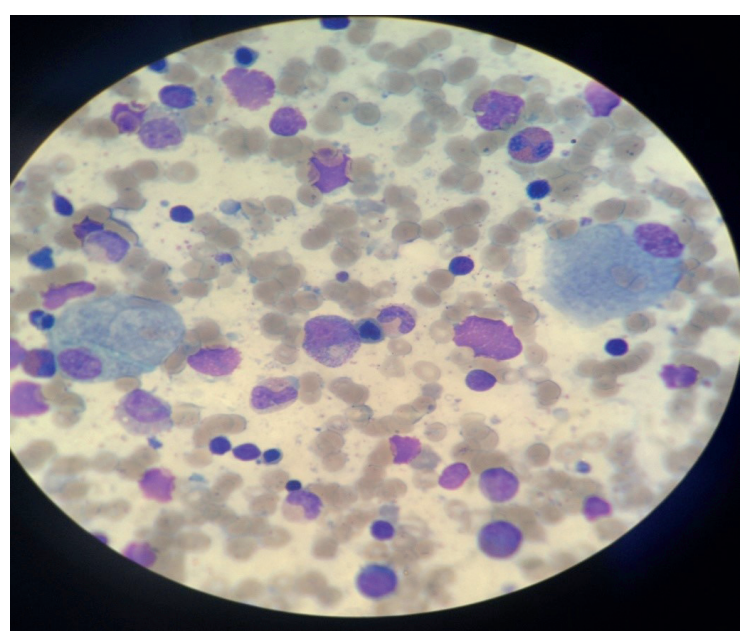

Fig. 1. Lipid-laden macrophages in bone marrow.

After the diagnosis of GD and initiating the enzyme therapy he took intra-arterial melphalan treatment for his left eye. Unfortunately, he was unresponsive to the treatment, thus enucleation was also performed on his left eye. $\mathrm{He}$ is presently alive and is still followed up with enzyme replacement therapy. Informed consent was obtained from the family before this case report.

\section{Discussion}

It has been reported that patients with GD have an increased lifetime cancer risk in particular of hematological origin. Shiran A et al. ${ }^{1}$ reported a 14.7-fold increased risk of hematologic malignancies. It has been speculated that the chronic stimulation of the immune system by excessive glucocerebrosides accumulation may cause lymphoproliferation. ${ }^{7}$ There are also several case reports of non-hematological malignancies reported in association with GD (including bone, colon, liver, lung, kidney, brain). ${ }^{8}$ Until now coexistence between GD and retinoblastoma has not been reported. Retinoblastoma is the most common primary intraocular malignancy of childhood. Seventy five percent of patients with retinoblastoma present with unilateral disease at a median age peak of 2 to 3 years. ${ }^{9}$ Bilateral cases tend to present at a younger age and have a mean age of onset 9 months earlier than unilateral cases. Our patient was diagnosed with bilateral retinoblastoma at the age of 2 months. Knudson's 2-hit hypothesis disclosed why the onset of bilateral retinoblastoma is earlier than the unilateral ones. He suggested that at least 2 mutations are required for tumor initiation; germline mutation and a mutation in any somatic cell and germline mutation do often portend an earlier presentation. ${ }^{10}$

Our patient was diagnosed with GD at the age of 11 months. The age of diagnosis depends substantially on the mutation that is detected in the patient. Mean age at diagnosis in the patients with the N370S/N370S genotype was 27.2 years (SD, 19.7 years); with L444P/L444P genotype, 2.3 years (SD, 3.2 years). ${ }^{11}$ The patient was homozygous for the L444P point mutation. L444P is the most prevalent mutation accounting for $42 \%$ in Turkish patients. ${ }^{12}$ To our knowledge L444P homozygosity tends to result in neuronopathic disease, although he had no neurological involvement maybe because of early age at diagnosis, the patient is most probably GD type 3 .

In the literature, there are few cases that report the co-existence of malignancies and GD in the pediatric age group. One report with 2 cases is about the association between the GD and acute lymphoblastic leukemia, the other one is a 16 year-old male with peripheral 
T-cell lymphoma. ${ }^{13,14}$ For non-hematological malignancies there is one report, an 18-yearold adolescent presenting with neuroblastoma who was later diagnosed with GD. ${ }^{15}$ In 2005 the outcomes of Gaucher Registry, the database of nearly 3000 patients with GD, revealed no cancer between 0 and 10 years. ${ }^{3}$ Despite considering the lack of medical records of some cases, on the basis of this comprehensive study, the diagnosis of cancer in children with GD seems to be quite rare.

There are some reports concentrating on the risk of cancer in GD with specific mutations. But there is no data, which has shown the genotype correlation with cancer. ${ }^{2}$

Several theories have been suggested to clarify the association between GD and cancer. Overemphasized theory focuses on the accumulation of glucocerebroside as the main factor impacting the immune system. Excessive accumulation of glucocerebroside exaggerates B-cell function, dysregulates T-cell function, disrupts the antigen presentation and triggers macrophage activation, leading to chronic stimulation of the immune system. ${ }^{16}$ This dysregulations result in enhanced cytokine secretion and B-cell expansion.

The other theory is the retention of bioactive lipids. Progressively accumulated lysosomal glucocerebroside is deacylated and transported out of the lysosomes. Glucocerebroside and glucosylsphingosine (deacylated glucocerebroside) are hydrolyzed by neutral glucoserebrodisidase in the extralysosomal compartment and generating bioactive lipids, ceramide, glucose and sphingosine. Sphingosine is phosphorylated via two kinases and this leads to the formation of sphingosine-1- phosphate, which has anti-apoptotic, pro-mitogenic and proangiogenic effects. ${ }^{17}$ The retention of mutant glucocerebrosidase molecules themselves in the endoplasmic reticulum may lead to the formation of harmful protein aggregates causing cellular toxicity. Splenectomy and hyperferritinemia are the other factors contributing to cancer development in GD. ${ }^{8}$ In our patient retinoblastoma developed at a rather young age and before the diagnosis of GD. It is highly suspicious that glucoserebroside accumulation induced immune dysregulation before clinically overt GD. Thus, we believe the co-existence of retinoblastoma and GD in our patient does not support the theories mentioned above and suggests that both diseases are coincidental.

In conclusion, we reported a male infant with two rare diseases, even rarer considering his age. Although the pathophysiology of the association between GD and cancer remains unclear and there are controversies concerning this association; malignancies should be kept in mind during GD patients follow up.

\section{REFERENCES}

1. Shiran A, Brenner B, Laor A, Tatarsky I. Increased risk of cancer in patients with Gaucher disease. Cancer 1993; 72: 219-224.

2. Taddei TH, Kacena KA, Yang $M$, et al. The underrecognized progressive nature of N370S Gaucher disease and assessment of cancer risk in 403 patients. Am J Hematol 2009; 84: 208-214.

3. Rosenbloom BE, Weinreb NJ, Zimran A, Kacena KA, Charrow J, Ward E. Gaucher disease and cancer incidence: a study from the Gaucher Registry. Blood 2005; 105: 4569-4572.

4. Zimran A, Liphshitz I, Barchana M, Abrahamov A Elstein D. Incidence of malignancies among patients with type I Gaucher disease from a single referral clinic. Blood Cells Mol Dis 2005; 34: 197-200.

5. Broaddus E, Topham A, Singh AD. Incidence of retinoblastoma in the USA: 1975-2004. Br J Ophthalmol 2009; 93: 21-23.

6. Kivela T. The epidemiological challenge of the most frequent eye cancer: retinoblastoma, an issue of birth and death. Br J Ophthalmol 2009; 93: 1129-1131.

7. Shoenfeld Y, Gallant LA, Shaklai M, Livni E, Djaldetti M, Pinkhas J. Gaucher's disease: a disease with chronic stimulation of the immune system. Arch Pathol Lab Med 1982; 106: 388-391.

8. Arends $M$, van Dussen L, Biegstraaten M, Hollak CE. Malignancies and monoclonal gammopathy in Gaucher disease; a systematic review of the literature. Br J Heamatol 2013; 161: 832-842.

9. Ortiz MV, Dunkel IJ. Retinoblastoma. J Child Neurol 2016; 31: 227-236.

10. Knudson AG Jr. Mutation and cancer: statistical study of retinoblastoma. Proc Natl Acad Sci U S A 1971; 68: 820-823.

11. Charrow J, Andersson HC, Kaplan P, et al. The Gaucher registry: demographics and disease characteristics of 1698 patients with Gaucher disease. Arch Intern Med 2000; 160: 2835-2843. 
12. Emre S, Gürakan F, Yüce A, Rolf A, Scott R, Ozen H. Molecular analysis of Turkish Gaucher disease patients: identification of novel mutations in glucocerebrosidase (GBA) gene. Eur J Med Genet 2008; 51: 315-321.

13. Sanchez R, Etzell J, Kim G, Packman S, Fairley C, Goldsby R. Pediatric malignancies: case 2. Peripheral T-cell lymphoma in an adolescent with unsuspected Gaucher disease. J Clin Oncol 2005; 23: 4792-4793.

14. Burstein Y, Rechavi G, Rausen AR, Frisch B, Spirer Z. Association of Gaucher's disease and lymphoid malignancy in 2 children. Eur J Haematol 1985; 35: 445-447.
15. Madeo A, Garaventa A, Sementa AR, Suffia C Di Rocco M. The unusual association between Neuroblastoma and Gaucher Disease: Case report and review of the literature. Blood Cells Mol Dis 2018; 68: 106-108.

16. Choy FY, Campbell TN. Gaucher disease and cancer: concept and controversy. Int J Cell Biol 2011; 2011: 150450 .

17. Mistry PK, Taddei T, vom Dahl S, Rosenbloom BE. Gaucher disease and malignancy: a model for cancer pathogenesis in an inborn error of metabolism. Crit Rev Oncog 2013; 18: 235-246. 